\title{
Monitoring the Effects of Anti-angiogenesis on the Radiation Sensitivity of Pancreatic Cancer Xenografts Using Dynamic Contrast-Enhanced CT
}

\author{
Ning Cao, Ph.D., \\ School of Health Sciences, Purdue University, 550 Stadium Mall Drive, West Lafayette, IN USA \\ 47907-2051. Radiation Oncology, University of Washington, 1959 NE Pacific Street, Seattle, WA, \\ USA, 98195, Phone: (206) 598-1638, Fax: (206) 598-6218
}

Minsong Cao, Ph.D.,

Radiation Oncology, University of California-Los Angeles, Department of Radiation Oncology, 200 UCLA Medical Plaza, Suite B265, Los Angeles, CA, USA 90095-6951, Phone: (310) 825-9775

Helen Chin-Sinex, B.S., Radiation Oncology, Indiana University School of Medicine, 535 Barnhill Drive, RT 041, Indianapolis, IN, USA 46202-5289, Phone: (317) 278-0404, Fax: (317) 278-0405

\begin{abstract}
Marc Mendonca, Ph.D., Radiation Oncology, Indiana University School of Medicine, 535 Barnhill Drive, RT 041, Indianapolis, IN, USA 46202-5289, Phone: (317) 278-0404, Fax: (317) 278-0405
\end{abstract}

Song-Chu Ko, M.D., Ph.D., and

Radiation Oncology, Indiana University School of Medicine, 535 Barnhill Drive, RT 041, Indianapolis, IN, USA 46202-5289, Phone: (765) 448-8000, Fax: (765) 448-7694

\section{Keith M Stantz, Ph.D.}

School of Health Sciences, Purdue University, 550 Stadium Mall Drive, West Lafayette, IN, USA 47907-2051, Radiology and Imaging Sciences, Indiana University School of Medicine, 950 West Walnut Street, Indianapolis, IN, USA, 46202-5289, Phone: (765) 496-1874, Fax: (765) 496-1377

Keith M Stantz: kstantz@purdue.edu

\section{Abstract}

\begin{abstract}
Purpose-To image the intra-tumor vascular physiological status of pancreatic tumors xenografts and their response to anti-angiogenic therapy using Dynamic Contrast-Enhanced CT (DCE-CT), and to identify parameters of vascular physiology associated with tumor X-ray sensitivity following anti-angiogenic therapy.
\end{abstract}

Methods and Materials-Nude mice bearing human BxPC-3 pancreatic tumor xenografts were treated with $5 \mathrm{~Gy}$ of radiation therapy (RT), either a low-dose $(40 \mathrm{mg} / \mathrm{kg})$ or a high-dose $(150 \mathrm{mg} /$ $\mathrm{kg}$ ) of DC101, the anti-VEGF receptor-2 anti-angiogenesis antibody, or with combination of low

\footnotetext{
(c) 2013 Elsevier Inc. All rights reserved.

Correspondence to: Keith M Stantz, kstantz@purdue.edu.

Conflict of Interest: None
}

Publisher's Disclaimer: This is a PDF file of an unedited manuscript that has been accepted for publication. As a service to our customers we are providing this early version of the manuscript. The manuscript will undergo copyediting, typesetting, and review of the resulting proof before it is published in its final citable form. Please note that during the production process errors may be discovered which could affect the content, and all legal disclaimers that apply to the journal pertain. 
or high dose DC101 and 5Gy RT (DC101-plus-RT). DCE-CT scans were longitudinally acquired over three week period post-DC101 treatment. Parametric maps of tumor perfusion and fractional plasma volume $\left(\mathrm{F}_{\mathrm{p}}\right)$ were calculated and their averaged values and histogram distributions evaluated and compared to controls, from which a more homogeneous physiological window was observed 1-week post-DC101. Mice receiving a combination of DC101-plus-RT(5Gy) were imaged baseline prior to receiving DC101 and 1-week after DC101 (prior to RT). Changes in perfusion and $\mathrm{F}_{\mathrm{p}}$ were compared with alternation in tumor growth delay for RT and DC101-plusRT(5Gy) treated tumors.

Results-Pretreatment with low or high doses of DC101 prior to RT significantly delayed tumor growth by an average 7.9 days compared to RT alone (p $₫$ ).01). The increase in tumor growth delay for the DC101-plus-RT treated tumors was strongly associated with changes in tumor perfusion $(\Delta \mathrm{P}>-15 \%)$ compared to $\mathrm{RT}$ treated tumors alone $(\mathrm{p}=0.01)$. In addition, further analysis revealed a trend linking the tumor's increased growth delay to its tumor volume-to-DC101 dose ratio.

Conclusions-DCE-CT is capable of monitoring changes in intra-tumor physiological parameter of tumor perfusion in response to anti-angiogenic therapy of a pancreatic human tumor xenograft that was associated with enhanced radiation response.

\section{INTRODUCTION}

Pancreatic cancer is the fourth leading cause of cancer death and the most lethal of all solid tumors, with an overall 5-year survival rate of less than 5\% [1]. To improve patient outcome, new therapeutic strategies are necessary. One such strategy is to combine new classes of targeted drugs to improve upon established treatment protocols, and to identify diagnostic parameters predictive of outcome using in vivo imaging techniques.

Studies have shown that pancreatic cancer is highly angiogenic with high levels of microvascular density and VEGF expression, which are correlated to metastasis and poor prognosis [2]. With the rapid development of anti-angiogenic drugs (AADs), a new genre of cancer treatment is now available. These anti-angiogenic inhibitors, such the anti-VEGFR2 monoclonal antibody DC101, have been shown to ablate tumor blood vessels resulting in hypoxia thus increasing radioresistance; however, they also can prune immature vessels and remodel tumor vasculature, thereby improving intra-tumor delivery of oxygen, reducing the number of cancer cells exposed to hypoxic conditions, and enhancing radiosensitivity [3]. We propose to identify an optimal dose of anti-angiogenic therapy and corresponding parameters of vascular physiology that may help improve tumor X-ray sensitivity following anti-angiogenic therapy. Previous reports have shown anti-angiogenic therapy can temporarily alleviate heterogeneous tumor vascular and blood flow in clinical and preclinical studies of colon cancer and glioma $[4,5]$, but, the appropriate protocol necessary to combine AADs to improve the therapeutic efficacy in pancreatic cancer remains unclear and requires further investigation.

To achieve an optimal outcome when combining anti-angiogenic therapy with RT, it is essential to track tumor angiogenesis in vivo pre- and post-anti-angiogenic therapy, in particular the structural and functional properties of the tumor vasculature. Dynamic contrast-enhanced CT or MRI (DCE-CT/DCE-MRI) can be used to monitor tumor physiology and its heterogeneity in response to AADs targeting the VEGF pathway [6, 7]. DCE-CT has an advantage of concurrently quantifying a number of vascular physiological parameters (perfusion; fractional plasma volume, $\mathrm{F}_{\mathrm{p}}$; permeability-surface area product, PS; and fractional interstitial space, $\mathrm{F}_{\text {is }}$ ) with high spatial resolution [7, 8]. Given this capability, DCE-CT is well suited to measure the small changes in tumor physiology consistent with vascular normalization. Previous studies have used DCE imaging to quantify these 
functional imaging biomarkers as early response factors to AADs $\left(\mathrm{F}_{\mathrm{p}}\right)$, therapeutic efficacy, and tumor cell proliferation and apoptosis (perfusion, vascularity or PS) $[9,10]$. To date, few studies have identified which of these diagnostic parameters and their intra-tumor distribution as a function of dose and time might relate to enhanced therapeutic efficacy to subsequent cytotoxic therapies in pancreatic cancer.

In this study, we investigated the dose-dependent physiological response to DC101 in pancreatic tumors, and subsequent changes in radiosensitivity. DCE-CT was used to monitor longitudinal changes in tumor physiology prior to and after DC101, from which a time to apply RT was determined. By investigating the relationship between these changes under different treatment conditions, a prognostic factor(s) associated with microvascular efficiency was identified as being associated with enhanced local tumor control.

\section{MATERIALS AND METHODS}

\section{Animal Model}

While under anesthesia (1.25\% isofluorane), $5 \times 10^{6}$ human pancreatic carcinoma BxPC-3 cells suspended in $0.1 \mathrm{ml}$ mixture (1:1 ratio) of phosphate buffered saline and Matrigel (BD Biosciences) were subcutaneously injected into the flanks of 6-8 weeks old female athymic (nu/nu) mice (Harlan Sprague-Dawley). Tumors were allowed to grow to $7-11 \mathrm{~mm}$ in size before treatment (Fig. S1). All animal preparation and procedures for this study were reviewed and approved by the Indiana University Institutional Animal Care and Use Committee.

\section{Experimental Design}

Sixty-eight mice with BxPC-3 pancreatic tumors were divided into a control group ( $\mathrm{n}=11)$; a DC101 therapy group consisting of a low-dose (LD) $(40 \mathrm{mg} / \mathrm{kg} ; \mathrm{n}=10)$ and a high-dose (HD) $(150 \mathrm{mg} / \mathrm{kg} ; \mathrm{n}=11)$ treatment; a RT group consisting of a 3, 5, and 7Gy treatment $(\mathrm{n}=4,12$, and 6 , respectively); and a combined DC101 and RT group consisting of a LD DC101-plus-RT(5Gy) group (LD-plus-RT; n=6) and a HD DC101-plus-RT(5Gy) group (HD-plus-RT; n=8). Each mouse in the DC101 groups was injected intraperitoneally (i.p.) with DC101 (a kind gift from ImClone, Inc.), and tumors for those mice in the RT group were exposed to X-rays with a dose rate of $1.6 \mathrm{~Gy} / \mathrm{min}$ (X-RAD 320 Biological Irradiator). In the LD and HD DC101 groups, DCE-CT scans were acquired within 24 hours of prior to DC101 injection, and 2, 7, 14, and 21 days after treatment. Mice receiving combined treatment had DCE-CT scans acquired at baseline (pre-DC101) and 1-week post-DC101 treatment. Immediately after this second scan, their tumor was treated with 5Gy of X-rays. To evaluate therapeutic response, the tumor growth delay curves for each experimental group were calculated by measuring the tumor volume every three days using calipers.

\section{Imaging Protocol}

Prior to DCE-CT imaging, each mouse was anesthetized with acepromazine and torbugesic $(0.1 \mathrm{mg} / \mathrm{kg}$, i.m.) and positioned within a clinical CT scanner (Brilliance, Phillips) with both the heart and tumor within the field-of-view $(40 \times 0.625 \mathrm{~mm}$ collimation). CT scans were acquired $(80 \mathrm{kVp} ; 80 \mathrm{mAs})$ prior to and after an intravenous injection of Isovue-370 (Bracco Diagnostic). Images were reconstructed with a voxel size of $0.3 \mathrm{~mm}$ in the transaxial plane and then be transferred to a sequence of workstations for further analysis $[7,8]$.

\section{Data Analysis}

Data analysis was performed as described in our previous publication [8]. First, physiological parametric maps (perfusion, $\mathrm{PS}, \mathrm{F}_{\mathrm{p}}, \mathrm{F}_{\mathrm{is}}$ ) were created by fitting the DCE-CT 
curves within each voxel to a two-compartmental mathematical model, from which the change in tumor physiology and intra-tumor heterogeneity was calculated. Next, the physiological value for each parameter was averaged over all voxels within each tumor, after which the fractional change in physiology, $\widetilde{\mathscr{T}}_{\mathrm{Q}}$, was calculated in order to minimize inter-animal variations: $\mathscr{F}_{Q}=\frac{Q i-Q_{\text {baseline }}}{Q_{i}}$, where i represents time (baseline, 2, 7, 14, and 21 days) and $\mathrm{Q}$ the physiological parameter (perfusion, $\mathrm{PS}, \mathrm{F}_{\mathrm{p}}, \mathrm{F}_{\mathrm{is}}$ ). The mean and standard deviation $(\mathrm{SD})$ of $\widetilde{\mathrm{Q}}$ was averaged over all mice in each group and compared to baseline values and across cohorts. Finally, the intra-tumor heterogeneity was assessed visually by inspecting the spatial variation of the parametric images over time (Fig. 2) and quantitatively by calculating the root-mean-square (RMS) of the histogram distributions for perfusion and $F_{p}$. For the latter, the RMS values were averaged over all mice in each group and its SD calculated at each time point.

\section{Doubling Times}

Tumor doubling times were determined by fitting the tumor growth curve for each mouse to the Gompertizian equation, a biology-based mathematical model of tumor growth [11]. The average (and SD) was calculated for each experimental group.

\section{Statistical Analysis}

One-way ANOVA and two-tailed Student's $t$ test were used to determine significant differences among groups. $P<0.05$ was considered statistically significant.

\section{RESULTS}

\section{Longitudinal Tumor Response to DC101}

The fractional changes in perfusion and $\mathrm{F}_{\mathrm{p}}$ relative to baseline values were averaged over all tumors in the control, HD or LD DC101 treated groups (Fig. 1). From these results, perfusion and $\mathrm{F}_{\mathrm{p}}$ in the control group remained relatively constant over time, with an overall decrease in $F_{p}$ primarily due to the formation of necrosis (Fig. S2). For DC101 treated tumors, an initial physiological response was observed within a couple of days post-DC101 injection, which was followed by the recovery of perfusion and $\mathrm{F}_{\mathrm{p}}$ over the next two weeks but at differing rates. The average change in perfusion, $\mathscr{T}_{\mathrm{P}}$, was significantly reduced in both HD and LD groups within 2-3 days after DC101 treatment compared to controls ( $-37 \%$ $(\mathrm{HD}) /-41 \%(\mathrm{LD})$ vs. $-0.6 \%(\mathrm{Ctrl}) ; \mathrm{P}<0.01)$ and remained below controls for the next two weeks, but recovered and exceeded control levels by third week $(-1 \%(\mathrm{HD}) / 24 \%(\mathrm{LD})$ vs. $-15 \%(\mathrm{Ctrl}) ; \mathrm{LD}, \mathrm{P}=0.11 ; \mathrm{HD}, \mathrm{P}<0.05)$. As for changes in $\mathrm{F}_{\mathrm{p}}\left(\tilde{\mathscr{T}}_{\mathrm{Fp}}\right)$, an obvious reduction was observed within 2-3 days after treatment compared to controls $(-22 \%(\mathrm{HD}) /-17 \%(\mathrm{LD})$ vs. $-6 \%(\mathrm{Ctrl}) ; \mathrm{HD}, \mathrm{P}<0.05 ; \mathrm{LD}, \mathrm{P}=0.08)$ and remained significantly below controls for only one week $(-28 \%(\mathrm{HD}) /-20 \%(\mathrm{LD})$ vs. $-6 \%(\mathrm{Ctrl}) ; \mathrm{P}<0.01)$ before recovering to control levels by week two. These results demonstrate a decoupling in time between perfusion and $\mathrm{F}_{\mathrm{p}}$ and thus a stepwise recovery of the tumor's vascular network and its functionality associated with the VEGF/VEGFR2 pathway in the BxPC-3 pancreatic tumors.

\section{Longitudinal Changes in Intra-tumor Heterogeneity}

Upon visual inspection, perfusion and $\mathrm{F}_{\mathrm{p}}$ parametric maps reveal an abnormal and heterogeneous vasculature characteristic of pancreatic tumors (Fig. 2) [12]. The level of heterogeneity (perfusion and $\mathrm{F}_{\mathrm{p}}$ ) is clearly visible $(0.03$-to- $0.5 \mathrm{~mL} / \mathrm{min} / \mathrm{mL})$, fluctuating over time (controls). To provide a quantifiable measure of intra-tumor heterogeneity [13], changes in the RMS for perfusion and $\mathrm{F}_{\mathrm{p}}$ histogram distributions (RMS-Perfusion and RMS$F_{p}$ ) were tracked over time (Fig. 1), where a significant decrease in RMS-Perfusion occurred 
1-week after DC101 treatment. RMS- $F_{p}$ remained unchanged. As a result, a more uniform and potentially efficient blood flow results, which is anticipated to improve oxygen delivery to the tumor [3]. Based on these results, the tumor vasculature is hypothesized to become temporarily homogenous 1-week post-DC101 treatment, at which time RT should be applied.

\section{Inhibition of Tumor Growth by Combining Radiation and Anti-angiogenic Treatment}

To evaluate whether pretreatment of anti-angiogenic therapy prior to RT can significantly improve the therapeutic response to X-ray radiation, the increase in tumor growth delay, defined as the in vivo tumor doubling time (table 1), was compared between control tumors, RT and combined RT-plus-DC101. For the latter, RT of 5Gy was chosen because it was the minimal dose resulting in a significant tumor growth delay (Fig. S3). To evaluate the effect of anti-angiogenic therapy on RT, the tumor growth curves for each individual tumor were analyzed beginning at the time of RT (1-week post-DC101) and compared to RT (5Gy) alone. Relative to control tumors, $5 \mathrm{~Gy}$ of RT increased tumor growth delay by 6.3 days (10.7 versus 17.0 days; $\mathrm{P}<0.01)$, as did $\mathrm{DC} 101$ alone $(\mathrm{P}<0.01)$. Tumors pretreated with either a low-dose or high-dose of DC101 prior to RT (5Gy) measured, on average, an increase in the tumor growth delay of 3.9 days, but did not reach significance compared to RT (5Gy) alone ( $\mathrm{P}=0.17$; one-way ANOVA). The doubling times for tumors receiving a combination of RT-plus-DC101, either a low-dose (21.0 \pm 7.8 days) or a high-dose (20.9 \pm 3.5 days) of DC101, had a similar growth delay ( $P>0.05$; two-tailed t-test), demonstrating a lack of DC101 dose dependency.

\section{Relationship between Changes in Tumor Physiology and Radiosensitivity}

Since the dose of DC101 did not provide an inhibitory advantage in tumor growth (Fig. 3), changes in perfusion and $\mathrm{F}_{\mathrm{p}}$ for these tumors were evaluated on an individual basis. We observed that tumors where both perfusion and $\mathrm{F}_{\mathrm{p}}$ were reduced post-DC101 (Fig. 4A) grew faster than those only receiving RT, while tumors maintaining their perfusion levels postDC101, in spite of a reduction in $F_{p}$, displayed an enhanced tumor delay and response to RT (Fig. 4B). Thus, perfusion was used to reclassify DC101-plus-RT mice as either negative or positive responders based on their change in tumor perfusion, less than or greater than $-15 \%$, respectively, one-week post-DC101. The percent reduction in perfusion for those positively responding tumors was significantly lower than those tumors that responded negatively (Fig. 5A; $\mathrm{p}<0.05$ ). $\mathrm{F}_{\mathrm{p}}$ significantly decreased in both groups, but was not prognostic. As shown in Fig. 5B and 5C, positive responders fell significantly below the RT (5Gy) growth curve (4 days after RT), and below tumors receiving 7Gy of radiation, while the negative responders fell above the RT (5Gy) growth curve (no delay). Unlike the low and high-dose groupings, the tumor doubling time for positive responders was $24.9( \pm 6.1)$ days, or 7.9 days more than RT (5Gy) cohort $(\mathrm{P}=0.012)$, while the doubling time for negative responders was $17.3( \pm 3.8)$ days, the same as $\mathrm{RT}(\mathrm{P}=0.86)$ (table 1$)$.

\section{DISCUSSION}

Currently, standard treatment for pancreatic cancer patients consists of surgery, chemotherapy (gemcitabine), radiation therapy, or a combination of these procedures. However, the role of RT remains unclear and controversial [14], leading to inconsistent use across clinics. Questions as to the appropriate dose and timing of RT and anti-angiogenic therapy have been suggested as a potential causal effect. Given that radio-resistance remains a major clinical problem in treatment response and failure in pancreatic cancer, monitoring tumor vascular physiology and hypoxia during therapy can provide important information to enhance local control [15] on an individual basis. In this study, we demonstrated that VEGFR-2 inhibition can favorably change the tumor microcirculation and subsequently 
enhance the radio-sensitivity in pancreatic tumors, and that functional imaging biomarkers associated with tumor microcirculation, in particular perfusion, can determine if enhanced radio-sensitivity has occurred.

DCE-CT was used to measure changes in the vascular physiologic parameters of BxPC-3 tumors after treatment with DC101 and to identify if an enhanced response to RT can be obtained, which to our knowledge, a yet unobserved method and effect in pancreatic cancer. Of the measured physiological parameters, perfusion was observed to be associated with tumor response to RT, and while $\mathrm{F}_{\mathrm{p}}$ did not, it did provide information on drug activity (Figs.4 and 5). By decreasing the $\mathrm{F}_{\mathrm{p}}$-to-perfusion ratio, a faster mean-transit-time was realized indicating a more efficient microcirculation and reduced hypoxia, resulting in a significant increase in tumor growth delay. This is consistent with several other studies, where a concomitant increase in perfusion and decrease in vascular volume increased tumor oxygenation post anti-angiogenic therapy, and when combined with chemotherapy or radiotherapy, significantly enhanced local tumor control [5, 16, 17]. For example, similar changes in vasculature and blood flow after DC101 treatment was observed to increase tumor oxygenation in U87 glioma xenografts [5]; in the vascular permeability-to-flow ratio $\left(\mathrm{K}^{\text {trans }}\right)$ and microvessel density after cediranib to identify vasculature normalization and patient outcome for glioblastoma patients [16]; and in vascular morphology, vessel leakage and permeability, and spatiotemporal distribution in perfusion to increase drug delivery in xenograft models of breast cancer [17]. These studies, as in this study, demonstrate a strong relationship between perfusion, vascular normalization, and hypoxia.

A limiting factor in the present study is the choice for the time at which to apply antiangiogenic therapy, 1-week prior to RT. We observed the intra-tumor heterogeneity appeared to be more uniform or homogenous one week after receiving DC101, and by the second week, this uniformity was lost, where tumor angiogenesis, neovascular growth, and vascular physiology was reestablished (Figs.1 and 2). Thus, the removal of neovasculature by DC101 may have temporarily alleviated heterogeneity in perfusion around 1-week post DC101. However, the radiation response at 1-week clearly varied across tumors, thus the results were inconclusive and the time unsubstantiated. Similarly, variations in the timing of anti-angiogenic therapy resulting in tumor vasculature normalization were observed in other models. In glioma xenografts, a normalization window occurred five days after DC101 treatment, resulting in a significant decrease in hypoxia and increase in tumor growth delay. However, this normalization window was short-lived, where the tumor response to radiation was significantly reduced during the first few days after DC101 and beyond 1-week [5]. Other AADs, such as sunitinib, when treating SCCVII xenograft tumors, observed an increase in $\mathrm{pO}_{2}$ within a range of days, 2-to-6 days, and extended tumor growth delay if RT was applied at an appropriate time [18]. The choice of a single time appears to be somewhat arbitrary. Further studies will need to be performed to map this therapeutic window over time, such as applying RT at different time points after DC101 treatment.

Under current anti-angiogenic therapy protocols, the applied dose is based on total body weight and a common time at which to apply RT (e.g., normalization) is anticipated. However, the inter-relationship of dose and timing introduces variability when combining anti-angiogenic therapy and RT under such protocols, as demonstrated in this study. Monitoring potential diagnostic factors such as perfusion or VEGFR-2 expression may help to reduce this uncertainty. In addition another potential diagnostic factor observed by Fenton et al is tumor volume, where they showed DC101-induced hypoxia in mammary carcinomas depended on the tumor size and DC101 dose [19]. In our study the (baseline tumor volume)to-(DC101 dose) ration or VDR was compared to the tumor's radiation sensitivity (tumor growth delay) and perfusion (Fig. S4). For the DC101-plus-RT cohort, tumor perfusion lying within a moderate VDR range $\left(3.16 \pm 1.28\left[\mathrm{~mm}^{3} /(\mathrm{mg} / \mathrm{kg})\right]\right)$ remained at their highest 
level (at 1-week) and was associated with an increase in tumor growth delay, while a VDR above or below this range did not respond or responded poorly to RT. Similar to Fenton et al, the appropriate dose may be related to the initial tumor burden and could help reduce the arbitrariness or uncertainty in the time at which a favorable response to radiation therapy. In our study, this relationship did not reach statistical significance and requires further study.

We observed improved tumor vasculature and blood flow after anti-angiogenic therapy in pancreatic cancer correlated with increased sensitivity to radiation. It is also possible that a concurrent reduction in survival mechanisms associated with the VEGF pathway may also contribute to the radio-sensitizing of the tumor, such as the indirect inhibition of vessel formation which can enhance the radiation effect under anoxia and hypoxia [20]; the downregulation of cell survival pathways can radiosensitize endothelial and cancer cells [21], the enhancement of tumor cell apoptosis due to the loss of endothelial cells derived paracrine factors [22]. Given that DC101 appears to remain active beyond 1-week (Fig. 1) while some tumors still respond negatively (Fig. 4) suggests improved microcirculation (and oxygenation) maybe a more prominent radiosensitive factor compared to inhibition of the VEGF pathway [5].

\section{CONCLUSIONS}

Overall, this study has shown the ability of DCE-CT in determining anti-angiogenic therapy related vascular normalization window for combined RT as well as identifying factors, in particular perfusion, that are associated with the response of pancreatic tumors to RT.

\section{Supplementary Material}

Refer to Web version on PubMed Central for supplementary material.

\section{References}

1. Saif MW. Anti-angiogenesis therapy in pancreatic carcinoma. J Pancreas. Mar 9; 2006 7(2):163-73.

2. Duffy JP, Eibl G, Reber HA, Hines OJ. Influence of hypoxia and neoangiogenesis on the growth of pancreatic cancer. Mol Cancer. 2003; 2:12-22. [PubMed: 12605718]

3. Jain RK. Normalization of tumor vasculature: an emerging concept in antiangiogenic therapy. Science. 2005; 307(5706):58-62. [PubMed: 15637262]

4. Tong RT, Boucher Y, Kozin SV, et al. Vascular normalization by vascular endothelial growth factor receptor 2 blockade induces a pressure gradient across the vasculature and improves drug penetration in tumors. Cancer Res. 2004; 64(11):3731-6. [PubMed: 15172975]

5. Winkler F, Kozin SV, Tong RT, et al. Kinetics of vascular normalization by VEGFR2 blockade governs brain tumor response to radiation: role of oxygenation, angiopoietin-1, and matrix metalloproteinases. Cancer Cell. 2004; 6(6):553-63. [PubMed: 15607960]

6. Gossmann A, Helbich TH, Kuriyama N, et al. Dynamic contrast-enhanced magnetic resonance imaging as a surrogate marker of tumor response to anti-angiogenic therapy in a xenograft model of glioblastoma multiforme. J Magn Reson Imaging. 2002; 15(3):233-40. [PubMed: 11891967]

7. Stantz KM, Cao M, Cao N, et al. Monitoring the Longitudinal Intra-tumor Physiological Impulse Response to VEGFR2 Blockade in Breast Tumors Using DCE-CT. Mol Imaging Biol. 2011; 13(6): 1183-95. [PubMed: 20957443]

8. Cao M, Liang Y, Shen C, et al. Developing DCE-CT to quantify intra-tumor heterogeneity in breast tumors with differing angiogenic phenotype. IEEE Trans Med Imaging. 2009; 28(6):861-71. [PubMed: 19150783]

9. Ren Y, Fleischmann D, Foygel K, et al. Antiangiogenic and radiation therapy: early effects on in vivo computed tomography perfusion parameters in human colon cancer xenografts in mice. Invest Radiol. 2012; 47(1):25-32. [PubMed: 22178893] 
10. Cyran CC, von Einem JC, Paprottka PM, et al. Dynamic contrast-enhanced computed tomography imaging biomarkers correlated with immunohistochemistry for monitoring the effects of sorafenib on experimental prostate carcinomas. Invest Radiol. 2012; 47(1):49-57. [PubMed: 21934514]

11. Demicheli R, Foroni R, Ingrosso A, et al. An Exponential-Gompertzian Description of LoVo Cell Tumor Growth from in Vivo and In Vitro Data. Cancer Research. 1989; 49:6543-6546. [PubMed: 2819710]

12. Schmidt J, Ryschich E, Daniel V, et al. Vascular structure and microcirculation of experimental pancreatic carcinoma in rats. Eur J Surg. 2000; 166(4):328-35. [PubMed: 10817332]

13. Sanghera B, Banerjee D, Khan A, et al. Reproducibility of 2D and 3D Fractal Analysis Techniques for the Assessment of Spatial Heterogeneity of Regional Blood Flow in Rectal Cancer. Radiology. 2012; 263(3):865-73. [PubMed: 22438361]

14. Hazard L. The role of radiation therapy in pancreas cancer. Gastrointest Cancer Res. 2009; 3(1): 20-8. [PubMed: 19343134]

15. Tredan O, Galmarini CM, Patel K, et al. Drug resistance and the solid tumor microenvironment. J Natl Cancer Inst. 2007; 99(19):1441-54. [PubMed: 17895480]

16. Sorensen AG, Batchelor TT, Zhang WT, et al. A "vascular normalization index" as potential mechanistic biomarker to predict survival after a single dose of cediranib in recurrent glioblastoma patients. Cancer Res. 2009; 69(13):5296-300. [PubMed: 19549889]

17. Zhang Q, Bindokas V, Shen J, et al. Time-course imaging of therapeutic functional tumor vascular normalization by antiangiogenic agents. Mol Cancer Ther. 2011; 10(7):1173-84. [PubMed: 21586628]

18. Matsumoto S, Batra S, Saito K, et al. Antiangiogenic agent sunitinib transiently increases tumor oxygenation and suppresses cycling hypoxia. Cancer Res. 2011; 71(20):6350-9. [PubMed: 21878530]

19. Fenton BM, Paoni SF, Ding I. Effect of VEGF receptor-2 antibody on vascular function and oxygenation in spontaneous and transplanted tumors. Radiother Oncol. 2004; 72(2):221-30. [PubMed: 15297140]

20. Lee CG, Heijn M, di Tomaso E, et al. Anti-Vascular endothelial growth factor treatment augments tumor radiation response under normoxic or hypoxic conditions. Cancer Res. 2000; 60(19):556570. [PubMed: 11034104]

21. Gupta VK, Jaskowiak NT, Beckett MA, et al. Vascular endothelial growth factor enhances endothelial cell survival and tumor radioresistance. Cancer J. 2002; 8(1):47-54. [PubMed: 11895203]

22. O'Reilly MS, Holmgren L, Chen C, Folkman J. Angiostatin induces and sustains dormancy of human primary tumors in mice. Nat Med. 1996; 2(6):689-92. [PubMed: 8640562] 
The role of radiation and anti-angiogenic therapy in pancreatic cancer remains controversial, where currently applied protocols are believed to contribute to the lack of any therapeutic benefit. After identifying tumor perfusion as a prognostic factor of radiosensitivity, the relationship between AAT dose and tumor burden on enhanced local control was investigated. 
A

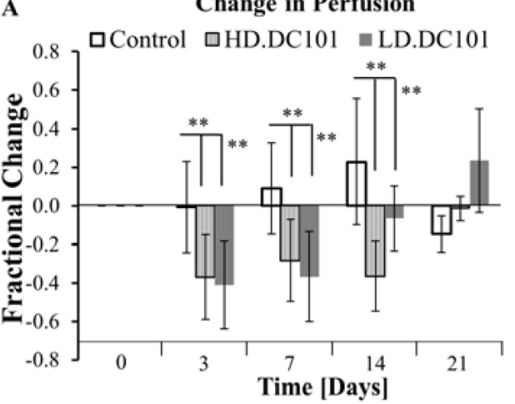

C

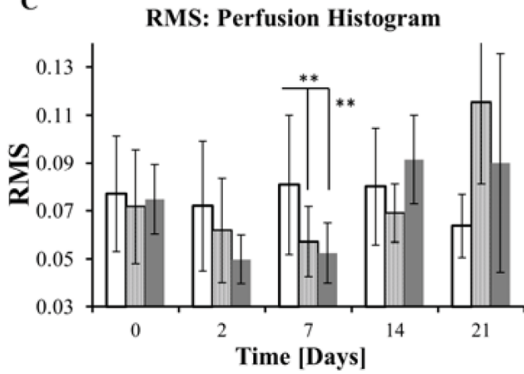

B Change in Fractional Plasma Volume

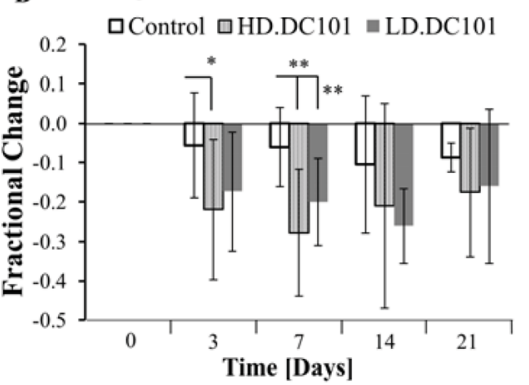

D

RMS: $\mathrm{F}_{\mathrm{p}}$ Histogram

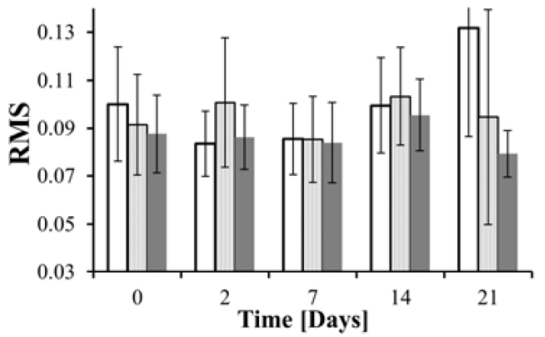

Fig. 1.

Plotted are the average percent change and standard deviation (SD) in tumor perfusion (A) and $F_{p}(B)$ as a function of time post-DC101 injection, and the average root-mean-square (RMS) of histogram distribution for perfusion $(\mathrm{C})$ and $\mathrm{F}_{\mathrm{p}}(\mathrm{D}) .(* \mathrm{P}<0.05$; ** $\mathrm{P}<0.01$, error bar: \pm SD) 


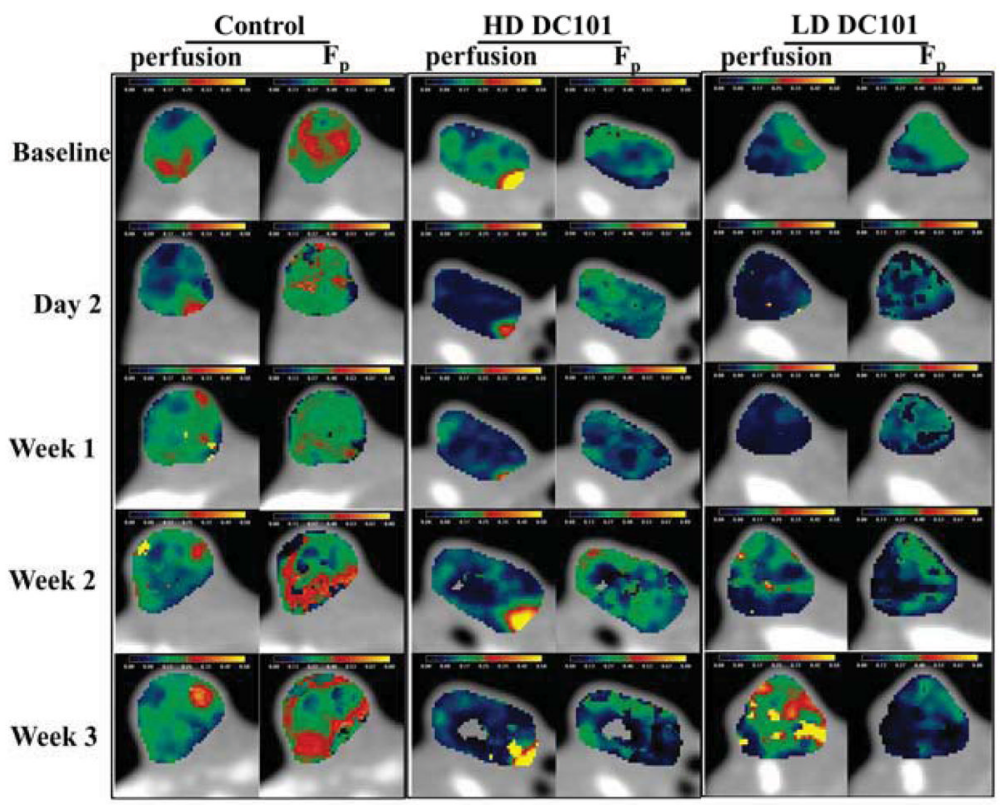

Fig. 2.

Physiological maps of perfusion and $\mathrm{F}_{\mathrm{p}}$ for $\mathrm{BxPC}-3$ pancreatic tumors at five different days: baseline, 2, 7, 14, and 21. From left to right: control, high-dose DC101, and low-dose DC101. 


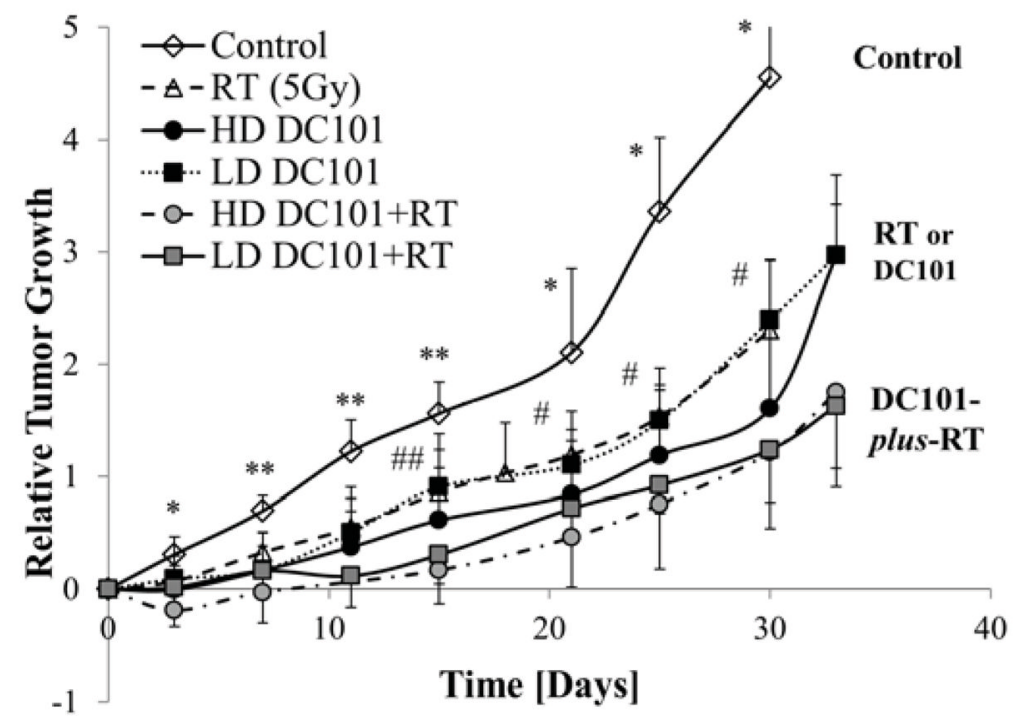

Fig. 3.

Plotted are the average relative tumor growth and SD as a function of time for control, highdose DC101, low-dose DC101, radiotherapy groups, and DC101-plus-RT (HD and LD) treatment groups $(* \mathrm{P}<0.05 ; * * \mathrm{P}<0.01$ among control and $\mathrm{RT}$ or $\mathrm{DC} 101$ groups; ${ }^{\#} \mathrm{P}<0.05$; ${ }^{\# \#} \mathrm{P}<0.01$, among DC101 and DC101-plus-RT groups; error bar: $\pm \mathrm{SD}$ ). 

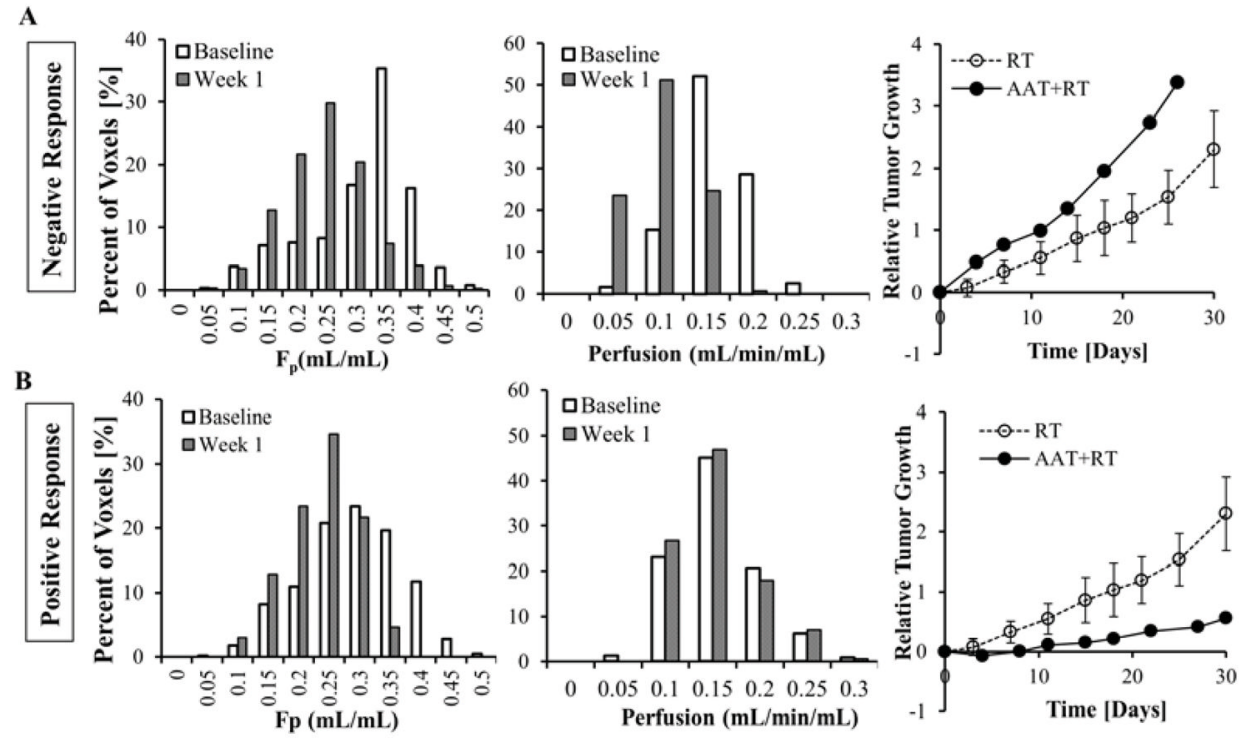

Fig. 4.

Tumors receiving combined DC101-plus-RT are categorized based on their physiological changes after DC101 treatment. Histograms for perfusion (left) and $\mathrm{F}_{\mathrm{p}}$ (middle), and relative tumor growth (right), for a representative tumor in the negative (A) and positive (B) responding groups are displayed. 

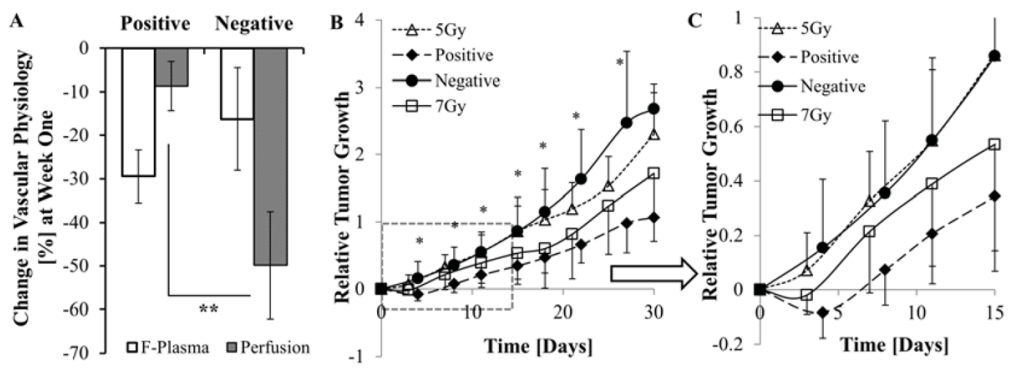

Fig. 5.

(A) Average percentage change in perfusion and $F_{p}$ for the positive $(n=4)$ and negative $(n=$ 5) responding tumors 1-week post-DC101 treatment are compared to corresponding baseline values. (B) Average relative growth in the positive and negative responding tumors is compared to 5 and 7 Gy RT groups. (C) Represents the portion inside the dashed line rectangular in Fig. 5B, which highlights the reduced variance and statistical significance in the early tumor growth delay response. $(* \mathrm{P}<0.05$; $* * \mathrm{P}<0.01$; error bar: $\pm \mathrm{SD})$ 
